\title{
DENOISING OF 3D POINT CLOUDS
}

\author{
E. Mugner ${ }^{1, *}$, N. Seube ${ }^{2}$ \\ ${ }^{1} 13$, Av de l'Europe, 31520 Ramonville Ste-Agne, FRANCE elliot.mugner@geown.io \\ ${ }^{2} 3051$ rue du Plateau, J7V 8P2 Vaudreuil-Dorion, Québec, CANADA - nicolas.seube@ geown.io
}

\section{Commission II}

KEY WORDS: Denoising, Airborne LiDAR, 3D point clouds

\begin{abstract}
:
A method to remove random errors from 3D point clouds is proposed. It is based on the estimation of a local geometric descriptor of each point. For mobile mapping LiDAR and airborne LiDAR, a combined standard mesurement uncertainty of the LiDAR system may supplement a geometric approach. Our method can be applied to any point cloud, acquired by a fixed, a mobile or an airborne LiDAR system. We present the principle of the method and some results from various LiDAR system mounted on UAVs. A comparison of a low-cost LiDAR system and a high-grade LiDAR system is performed on the same area, showing the benefits of applying our denoising algorithm to UAV LiDAR data. We also present the impact of denoising as a pre-processing tool for ground classification applications. Finaly, we also show some application of our denoising algorithm to dense point clouds produced by a photogrammetry software.
\end{abstract}

\section{INTRODUCTION}

Point clouds acquired by low-cost UAV LiDAR or Photogrammetric systems are, for some of them, subject to a significant level of uncertainty. Combined with relatively low sampling rates in the case of LiDAR data, this may cause difficulties to reconstruct accurately underlying surfaces or $3 \mathrm{D}$ structures.

Three types of errors may affect the uncertainty of LiDAR point clouds:

- Systematic errors generate uncertainty that may become visible within overlaps;

- Random errors from sensors (IMU, GNSS, LiDAR) produce measurement noise;

- Non modeled errors generate outliers.

In this paper, we shall focus on techniques to remove the random measurement noise that may degrade $3 \mathrm{D}$ point clouds precision.

Most denoising methods apply filters that remove the high frequency component of a local surface (Rosmanand et al., 2013), at the price of smoothing irregularities and 3D features. One of the main issues of denoising is the design of a method that both reduce the level of noise while preserving details and features that the user wants to recover from the LiDAR or the photogrammetric point cloud. Buildings, as all man-made infrastructures (pylons, power lines, dams, etc...), are present in point clouds. As they naturally exhibit edges, the main requirement of a denoising algorithm is to preserve them.

The denoising method developed by GEOWN aims at reducing the level of random errors ${ }^{1}$ present in a point cloud. Random errors produce noise whilst gross errors produce outliers that no physical model can explain.

\footnotetext{
* Corresponding author

${ }^{1}$ Random errors should be distinguished from systematic errors and gross errors. Indeed, systematic errors are produced by the presence of
}

\subsection{Related work}

Several methods for denoising were proposed and most of them use the underlying local geometry of the point to be denoised (Deschaud, Goulette, 2010),(Duguet et al., 2004),(Digne, 2012). Most methods differ in the geometric models and modeling methods they use. For instance, the local normal vector to a point, estimated by covariance analysis (Boulch, Marlet, 2012),(Mitra, Nguyen, 2003) Randomized Hough Transform or Moving Least Square (Fleishman et al., 2005) may be used to describe the underlying local surface at a given point. Bilateral filters move points along the local normal to the surface, according to a weighting scheme defined as a function of the orthogonal distance of neighboring points to the normal. Descriptors of the point geometry can also be constructed to define a measure of similarity between the point and local smooth surfaces (Digne, 2012). Another method is based on Statistical Outlier Removal (SOR) filters which eliminate points with low local density, independently of the local surface. In the SOR-OD method (OD standing for Orthogonal Distance), the distance to neighbors is replaced by the orthogonal distance to a best fitted plane within the neighborhood of the point.

Most of these methods were designed for point clouds of quite different nature than the ones we experience in the UAV LiDAR industry. Indeed, most of the work on denoising was devoted to extremely dense data sets, at very low scale (scans of manufactured objects for instance). However, most UAV LiDAR produce point clouds at relatively low density, unlikely larger than $200 \mathrm{pts} / \mathrm{m} 2$. In this context, the techniques presented above are, for some of them irrelevant, as the construction of a local descriptor of the surface is unstable in the case of point clouds with low density and high level of noise.

bias in some integration parameter (boresight, latency, lever-arm) or by a sensor measurement bias (LiDAR range, for instance). Systematic errors produce inconsistencies in a point cloud formed by the superimposition of survey strips with overlaps, as produced by an UAV. 


\section{THE GEOWN APPROACH TO DENOISING}

\subsection{A geometric approach}

The GEOWN denoising tool is based on the estimation of a geometric descriptor of the local surface to each point to be denoised. Normals ${ }^{2}$ to the points are estimated and refined to preserve the local geometric features of the point cloud.

The local descriptors are generally determined by using a purely geometrical description of the neighborhood of each point. In case of very dense point clouds, this option is generally satisfactory, but for sparse point clouds (like the ones provided by $\mathrm{UAVs}$ ), it is relevant to take into account not only the local geometry, but also an uncertainty model of each point.

Besides, in a geometric filter approach, each point is considered with a unit weight, independently of its uncertainty level. Thus, if in the same neighborhood, some points are from overlapping and noisy strips (or from outer beams), they are denoised together with points of better quality. The overall result will be an unweighted average between good quality and a low quality points, which may create a bias in the final denoising result.

To overcome these difficulties, we developed a combined geometric/probabilistic approach.

\subsection{A Combined Standard Measurement Uncertainty (CSMU) estimation}

To estimate the uncertainty associated to each LiDAR point that will be used in our probabilistic denoising method, we developed a combined standard measurement uncertainty model. The first stage is to make a first order approximation of the classical point georeferencing model, obtained from the geometry and timing model of the LiDAR system. The geometry of a LiDAR system (see figure 1) is defined through a Local Geodetic Frame (navigation frame $((n))$, used for orientation purposes, the choice of Positioning Reference Point (PRP, denoted by $P_{n}$ ) (i.e; the point at which position is computed by the GNSS system), the lever-arm vector $a$ from the PRP to optical center of the LiDAR and the boresight between the INS frame and the LiDAR body frame.

We write the georeferencing model as follows:

$$
X_{n}(t)=P_{n}\left(t-d t_{p}\right)+C_{b I}^{n}\left(t-d t_{i}\right)\left(C_{b S}^{b I} r_{b S}(t)+a_{b I}\right)
$$

where $t$ is the time associated to the LiDAR data, $d t_{p}, d t_{i}$ are respectively the time delays from LiDAR to positioning and INS. $C_{b I}^{n}$ is the navigation frame to boty INS transformation, and $C_{b S}^{b I}$ is the boresight transformation.

We consider variations of the point geo-referencing model given by equation (1) due to these input parameters variations:

- Time-stamping may be subject to delays (position-LiDAR and IMU-LiDAR), denoted by $d t_{p}$ and $d t_{i}$

- $P_{n}, a_{b I}$

- $(\varphi, \theta, \psi)$ defining the IMU to navigation frame transformation $C_{b I}^{n}$,

${ }^{2}$ The normal to a point $p$ can be computed by a Principal Component Analysis of the neighbors of $p$ in using their covariance matrix.

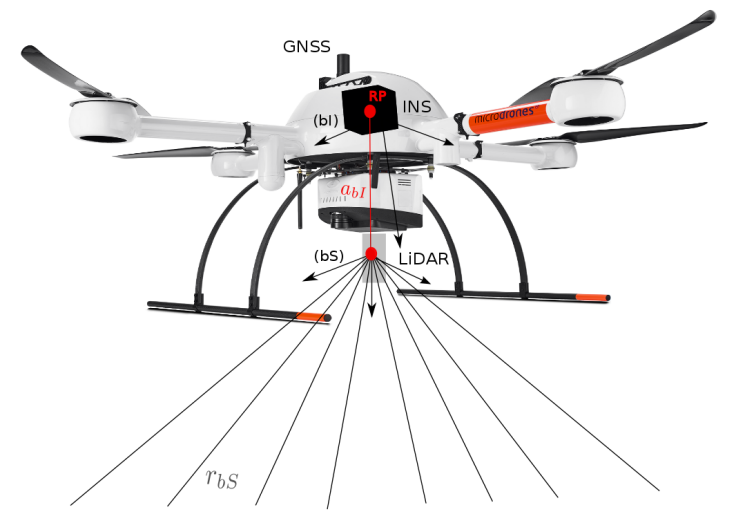

Figure 1. Geometry of an airborne LiDAR system. The positioning system is supposed to deliver the position of the PRP, denoted by $P_{n}$. The lever-arm is denoted by $\vec{a},(b S)$ is the LiDAR body frame, and $(b I)$ is the IMU body frame.

- $\left(\varphi_{b}, \theta_{b}, \psi_{b}\right)$ defining the LiDAR to IMU mis-alignment $C_{b S}^{b I}$.

- $\rho, \alpha, \beta$ defining the LiDAR return $r_{b S}$.

To supplement this error model, we introduced the grazing angle between the LiDAR beam and the local terrain, which introduces a range bias due to the other variables (attitude, boresight, position, lever-arm, latency). This CSMU uncertainty model is given as a covariance matrix $\Sigma_{\delta X_{n}}$ associated to each point. A result of this model is shown in figure (2).

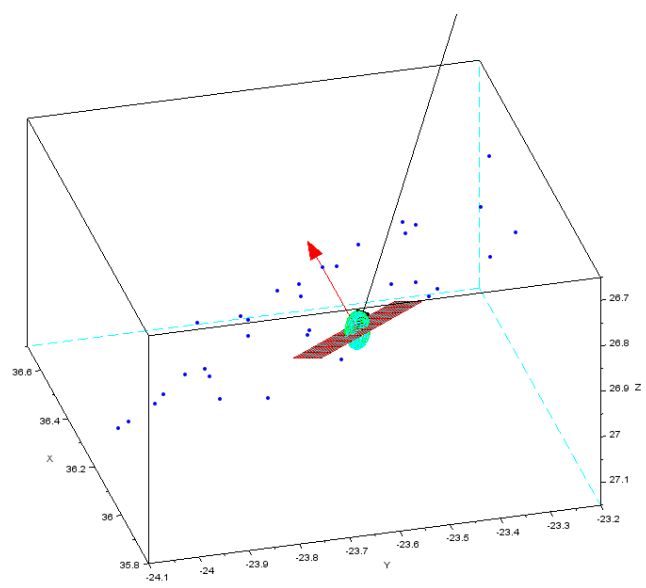

Figure 2. The uncertainty ellipsoid associated to a LiDAR point, as defined by our CSMU model including grazing effects. The local plane to the point and its normal are displayed. The straight line is the line from the LiDAR to the point. One can see that the ellipsoid main axis is relatively close to this axis.

\subsection{The GEOWN probabilistic denoising approach}

The approach proposed by GEOWN provides to the user a tool to reduce the noise level of an UAV LiDAR point cloud while preserving sharp edges and features. To do so, we introduced a denoising filter including both a geometrical and a probabilistic analysis, as explained below.

Suppose that we can associate to each point its covariance matrix and therefore its confidence ellipsoid representing its Combined Standard Measurement Uncertainty (CSMU) model. The 
CSMU is computed by using the estimated standard uncertainty of each sensor (GNSS, IMU, LiDAR), as explained in the previous section. For the LiDAR system, ranging uncertainty and scan angle precision are generally given by the manufacturer.

Whenever this model is representative of the point actual uncertainty, we can use it to make a compromise between the local geometry and the minimization of the point uncertainty level. Indeed, the confidence ellipsoid allows the algorithm to extend a point coordinates to a multiplicity of probable positions that can be used to accurately estimate the true coordinates of the point.

The GEOWN probabilistic filter generalizes geometric approaches at two different levels:

- The displacement step is weighted by the CSMU of its neighbors. If the neighbors to a point exhibit different levels of uncertainty, then the displacement step associated with this point will be more impacted by points that have a low level of uncertainty. By doing so, our method acknowledge the fact that all points of a point cloud are not sharing a common confidence level concerning the geometrical descriptors that can be computed from their coordinates.

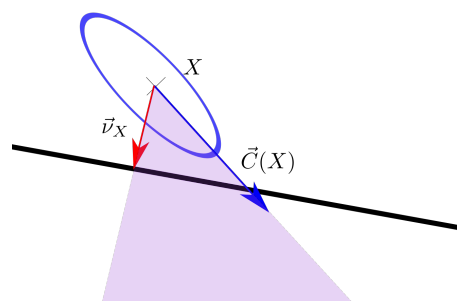

Figure 3. A point $X$, its ellipsoid of uncertainty, its normal vector to the local plane $\vec{\nu}_{X}$. Classical algorithms move points along $\vec{\nu}_{X}$. The GEOWN algorithm makes a compromise between $\vec{C}(X)$, the semi-major axis of the uncertainty ellipsoid and the normal $\vec{\nu}_{X}$. The denoised point will be located within the cone formed by these two vectors, making a geometric and probabilistic compromise.

- Instead of being the normal vector to the current point $p$, the displacement vector is a direction optimizing the probability level of the displaced point together with a geometrical criterion defined by a local plane (Cf. Figure 3 ).

This geometric/probabilistic approach makes a fundamental difference between our denoising algorithm and the purely geometrical approaches that were proposed in the literature. By taking into account the acquisition conditions of each point, our algorithm respects the raw data associated to the points and compute an estimation of the denoised positions that reflects these raw data. As shown on the Figure (4), the knowledge of the CSMU is an information reflecting the conditions in which a point has been acquired; and the estimation of a denoised position guided only by the normal vector of the scanned surface could lead in many cases to a denoised point that is no longer coherent with its own raw data.

Finally, the GEOWN probabilistic approach to point cloud denoising overcome the presence of outlying points near the

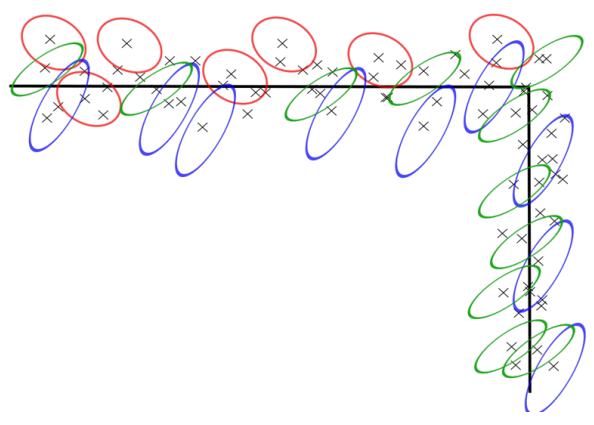

Figure 4. The knowledge of the acquisition history of each point allows the GEOWN denoising algorithm to estimate the denoised position of each point with respect to its acquisition history.

scanned surface. For some classical denoising methods, outliers ${ }^{3}$ can cause some artifacts by corrupting the surface normal estimation, leading to false denoised positions. The fact that each point CSMU is taking into account while estimating the position and orientation of the scanned surface allows the GEOWN denoising tool to eliminate points exhibiting an uncertainty ellipsoid largely incoherent with their neighbors.

The fact that our denoising method produces an estimation of each point denoised position coherent with the raw data allows us to propose a denoised position for almost all the elements of the point cloud. As a consequence, the output point cloud has the same density as the input one (where most popular denoising methods are removing points from the dataset). For applications that require to have a perfect match between the raw data and the point position, our tool is also capable to produce a denoised point cloud by suppressing points that are far from the estimation of their denoised position. By applying this second type of filter, the denoised point cloud has a lower density but the remaining points has not been moved at all.

\subsection{The GEOWN denoising workflows}

GEOWN developed two workflows that can adapt themselves to any point cloud:

1. For a general point cloud (from LiDAR or Photogrammetry), without LiDAR raw data: we apply a series of geometric filters that produce a denoised point cloud in the same coordinate system than the original one. The lack of knowledge about the points acquisition history does not allow our probabilistic tool to be used, but the combination of successive geometrical tools allows the computation of a denoised point cloud preserving sharp edges and sparse elements.

2. For LiDAR only and with raw data information, we first compute a CSMU model that feeds a series of geometric/probabilistic filters. This workflow is recommended for low precision LiDAR systems as the characteristics of each sensor are taken into account during the process.

\footnotetext{
${ }^{3}$ Outliers are not meant to be filtered by a denoising tool as they can not be considered as random noise. However, they can deeply impact the results of a denoising tool if their influence if not denied properly.
} 


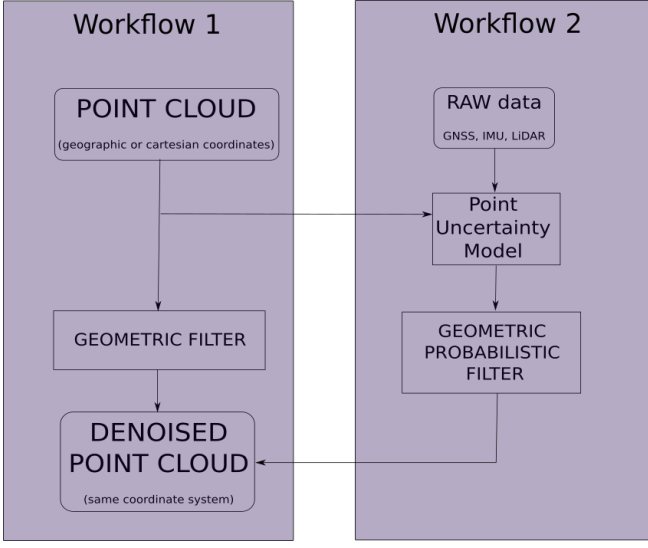

Figure 5. GEOWN denoising workflow.

\section{RESULTS OF DENOISING FOR POINT CLOUDS GENERATED BY A UAV LIDAR SYSTEM}

We provide some examples of results given by the GEOWN denoising algorithm. The point clouds that we used come from various LiDAR systems (Sick, Velodyne) integrated on UAVs. In this case, the typical initial point cloud thickness is around $15 \mathrm{~cm}$.

We start with a point cloud representing a building and surrounding ground (see Figure 6). This point cloud is challenging because it contains various elements such as regular ground and complex sharp edges. On this Figure, we see that the flat ground has been strongly denoised, while the roof has been lightly denoised in order to preserve the object characteristics.

Figure (7) illustrates how the denoising method behaves on an irregular curved object, without sharp edges but with small curvatures. To do this, we used a point cloud acquired on a car with a low cost UAV LiDAR system. We see that the car's structure has been recovered without artifacts. We remark that the interface between the different parts of the car are still smooth.

In Figure (8), we show the result of our denoising algorithm on a dataset produced by a Velodyne VLP16 coupled to an Applanix APX15. This kind of system exhibits a relatively high level of measurement noise that can be significantly reduced thanks to a GEOWN algorithm.

\section{COMPARISON WITH OTHER DENOISING METHODS}

In this section, we compare the GEOWN denoising algorithm with a Statistical Outlier Rejection (SOR) method using the Orthogonal Distance (OD) to local fitted planes. We shall denote this method as SOR-OD and see through a series of examples that it produces significant distortions on edges. This will illustrate the fact that the normal to a point is not necessarily the best direction to choose for denoising. This is a good reason to use the GEOWN denoising algorithm.

\subsection{Comparison for simulated point clouds}

To compare the two denoising methods, we simulated several point clouds containing elements that can be challenging for a denoising method and thus they can enlighten performance differences (sharp edges, curves, multiple surface orientations...).
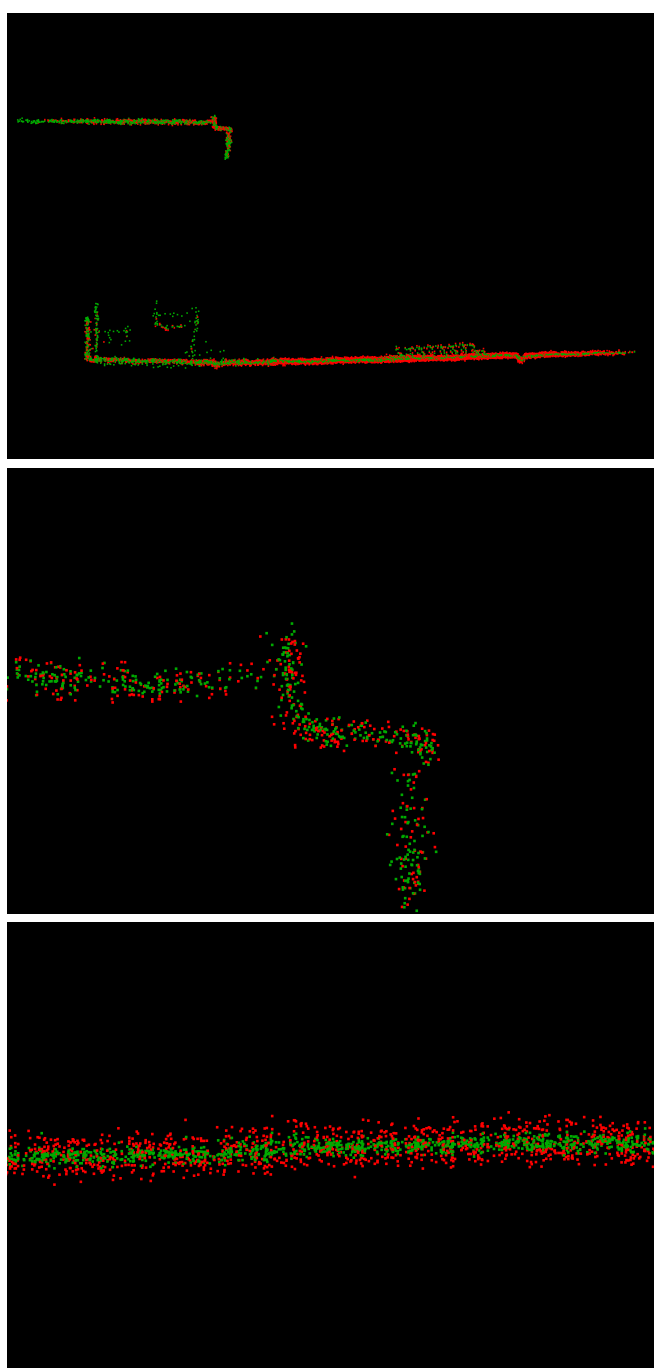

Figure 6. Denoising results on building (original point cloud in red, denoised point cloud in green). The two bottom images are

zooms respectively on the edge of the roof and on the flat ground. The results show that our method produced an accurate denoised point cloud respecting the underlying structures. Roof edges have been preserved while the ground has been significantly denoised. 

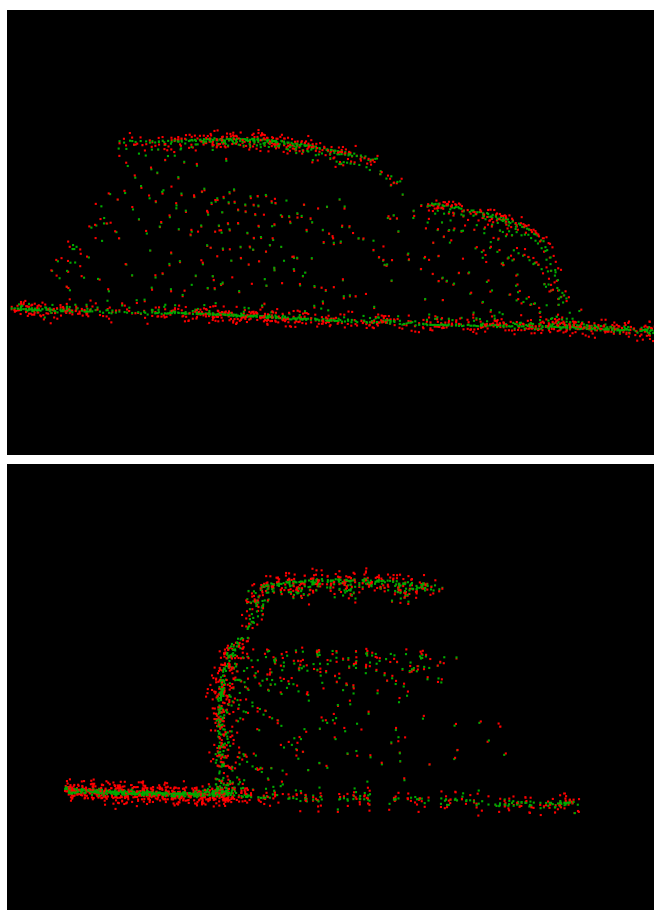

Figure 7. Denoising results on a car (original point cloud in red, denoised point cloud in green).

Figure (9) shows the denoising results for SOR-OD and for GEOWN's method on one of these artificial datasets. It can be seen that the trueness of GEOWN denoising is better than the trueness of the point cloud created by SOR-OD, when the two methods are applied with their optimal parametrization. The edge of the bi-plane is smoothed by the SOR-OD and the resulting denoised point cloud exhibits a delimitation reflecting the fact that the noisy points has been deleted with respect to their orthogonal distance to the scanned surface. On the other side, the denoised point cloud produced by the GEOWN denoising tool reflects the fact that an estimation of the denoised position of each point is computed, with respect to their raw data.

\subsection{Comparison for real point clouds}

Figure (10) illustrates GEOWN denoising and the SOR-OD denoising on a flat point cloud obtained by a SICK LiDAR coupled with an APX-15 INS mounted on a UAV. The thickness of the denoised point cloud produced by our tool is half the one of the point cloud denoised by the SOR-OD. This gain is allowed by the fact that our method replaces the points position by their denoised coordinates.

As mentioned before, one of the main characteristics of GEOWN's denoising method is to preserve sharp edges. Figure (11) illustrates the two methods for a building cornice using similar parameters for both methods. We observe that the SOR-OD method is suppressing some relevant points located at the limit of the cornice. However, these points contain essential geometric information about the shape of the cornice. The SOR-OD method thus appears to be unable to differentiate them from the rest of the cornice.
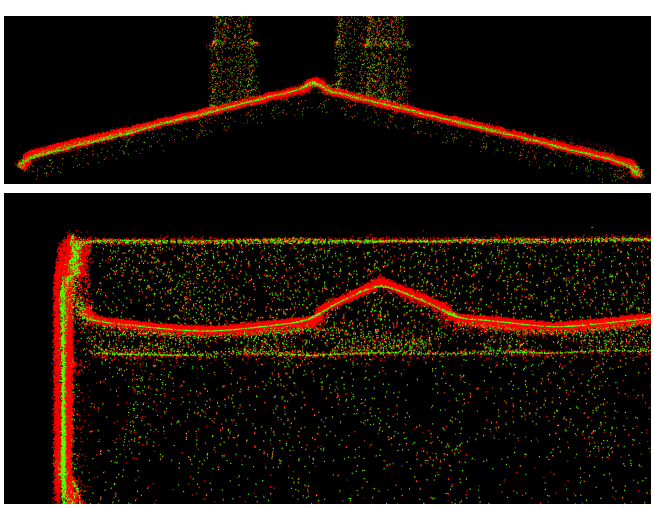

Figure 8. Denoising of a Velodyne VLP16 LiDAR on a wall and a roof (original point cloud in red, denoised point cloud in green). We can see that edges were preserved while walls and flat roof facets are strongly denoised.

\section{HOW DENOISING IMPROVES THE LIDAR SYSTEM PRECISION}

We show through examples that the GEOWN denoising tool can be used to enhance point clouds produced by many LiDAR sensors. This may be very interesting to reduce the gap between the original quality of the low precision LiDAR point cloud and the type of results that could be obtained by using high precision sensors.

In order to make a comparison, we are going to compare a point cloud from a Sick/APX-15 LiDAR, which produces point clouds with a thickness of about $15 \mathrm{~cm}$ and an other point cloud acquired with a Riegl VUX-1DL.

In Figure (12), we observe that the roof point cloud using the low precision LiDAR (in red) has a thickness of $15 \mathrm{~cm}$ which is 3 times the thickness of the high precision LiDAR point cloud. In addition, the two tiles on the roof are barely visible in the low-cost point cloud.

By applying GEOWN denoising, we can reduce the noise level of the low precision point cloud to match the thickness of the precise LiDAR. Figure (12) shows the result we obtained. The thickness of the denoised point cloud is close to the high grade point cloud and the sharp edge has been preserved. We also remark that the two tiles were preserved.

This first result shows how GEOWN denoising can improve the precision of a LiDAR system. Our algorithm can also be tuned according to the needs of the user: Figure (13) shows that if we require a very thin point cloud, the point neighbor size can be adapted accordingly. Here, the thickness of the low precision point cloud was reduced to a level below the noise level of the high grade LiDAR one.

The aim of a denoising method is to extract as much as possible information from the point cloud and to use this information to adjust the denoising to match the underlying structure of the scanned scene. The denoising method developed by GEOWN fulfills this task.

\section{RESULT FOR POINT CLOUD FROM PHOTOGRAMMETRY}

The behavior of a denoising algorithm is naturally improved in the case of high density point clouds, such as those produced 

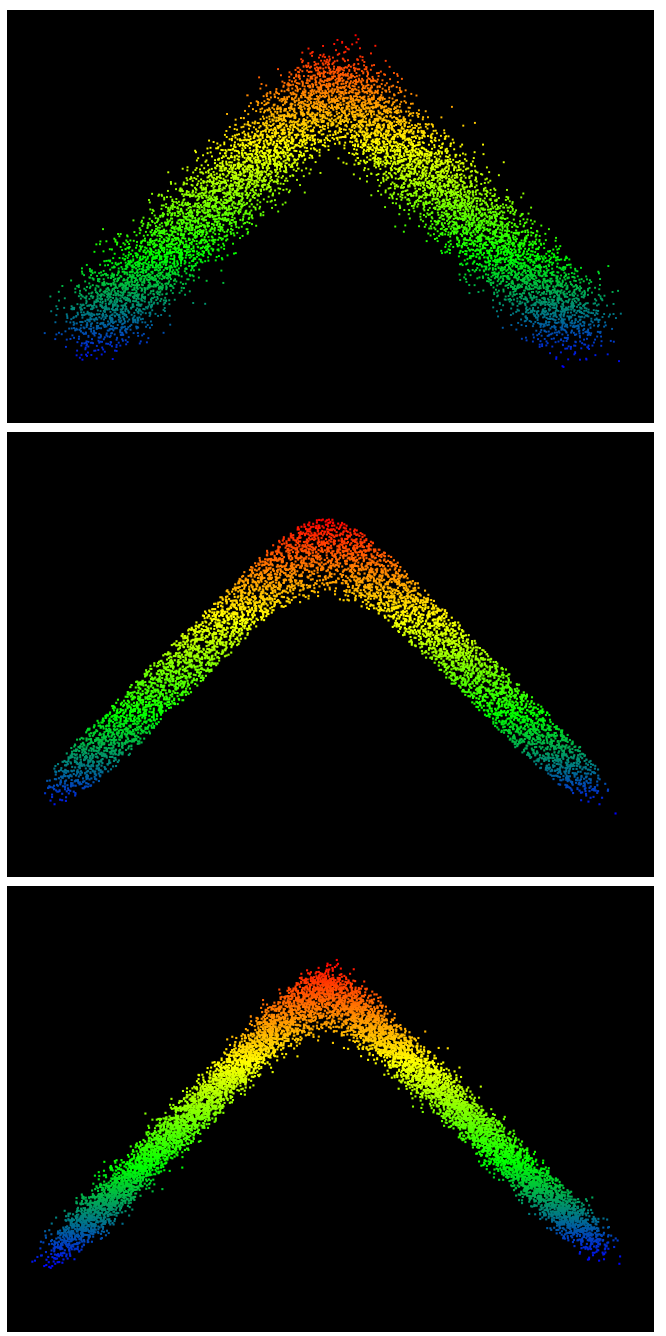

Figure 9. Denoising results on bi-plane with $30 \mathrm{~cm}$ radius (Original point cloud on top, reference denoising method on the middle and GEOWN on the bottom)

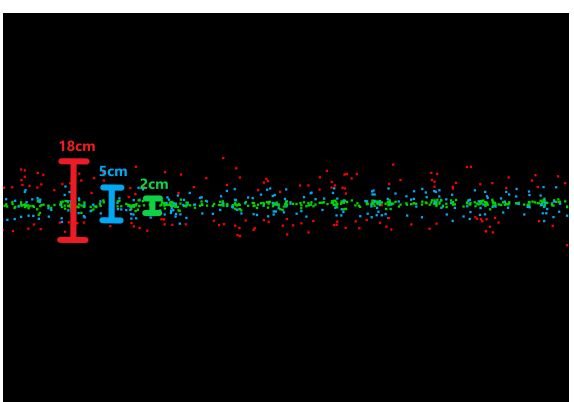

Figure 10. Denoising on flat surface (red) with classical filter (blue) and GEOWN filter (green).The two algorithms have been tuned with similar parameters: the size of the neighbourhood of each point was exactly of the same size in the two algorithms $(\mathrm{r}=50 \mathrm{~cm})$. One can easily check in this figure that we obtain a thickness of $2 \mathrm{~cm}$ against $5 \mathrm{~cm}$ for the SOR-OD.

by a photogrammetry process. Indeed, with a greater density, the local geometry of the point cloud can be estimated at lower scale, resulting in a better local geometric description of the point cloud.

To illustrate this fact, we tested the GEOWN denoising on a dense data set, produced by Fast ORtho Mapping (FORMap), a photogrammetry tool developed by GEOWN. The Ground Sampling Distance (GSD) of the dataset was $1 \mathrm{~cm}$. In Figure (14) top, a colorized point cloud from FORMap is shown. The car roof and hood are subject to typical errors of a photogrammetry process on sleek surfaces (lack of correlation between pixels) producing significant errors and noise. After denoising, we can see that the low GSD of the point cloud allows us to estimate accurate surface descriptors that can efficiently denoise the point cloud. Note that here, a purely geometric filter has been applied as the LiDAR system CSMU model can not be applied to photogrammetric datasets.

\section{THE IMPACT OF DENOISING ON LIDAR CLASSIFICATION TOOLS}

Point cloud denoising, unless of outliers detection, is an operation that is not largely used in classical point cloud processing workflow. This is mainly due to the fact that denoising is perceived as an operation of point cloud enhancement that is not necessary when the final usage of the point cloud is not visibly linked with the point cloud thickness. However, the denoising is actually a step that enhance, not only the point cloud characteristics, but also the accuracy and efficiency of other processing tools.

This section illustrates how the GEOWN denoising tool can improve the accuracy of a basic ground classification tool from LASTools (lasground). To do so, we compare the results of the ground classification on a raw point cloud acquired with a high quality LiDAR mounted on a UAV, and the results obtained with the same parametrization on the denoised point cloud. Figure (15) shows the two classified point clouds. We see that on the raw point cloud, the ground classification algorithm is not able to recover all the ground points. The noisy points are causing inaccurate normals estimations and thus erroneous orthogonal distance and slope estimation. As a consequence, the classified point cloud contains a lot of misclassified ground points.

The classification results obtained on the denoised point cloud are more accurate, because the reduced thickness of the point 

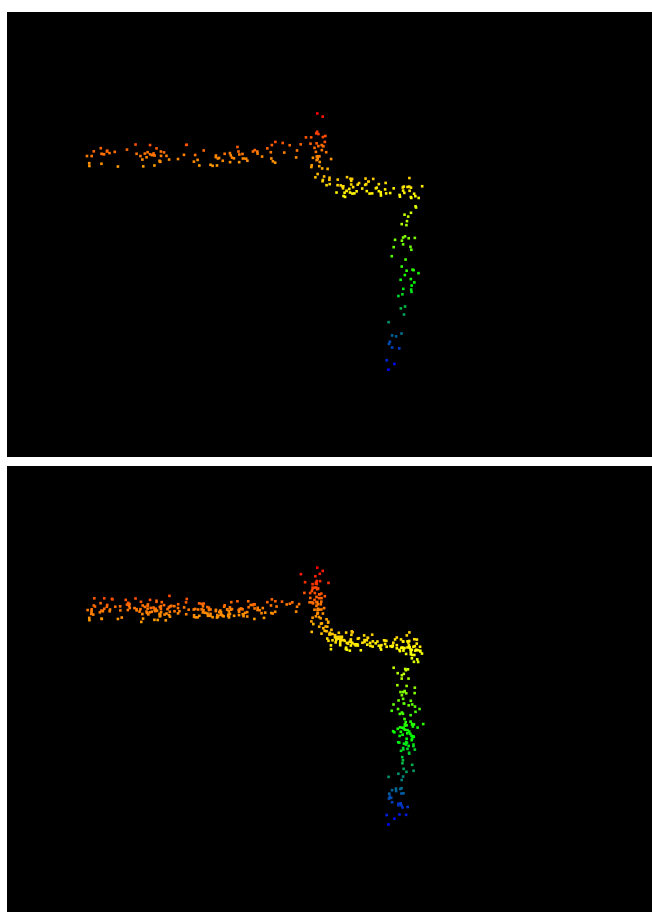

Figure 11. Denoising results on roof cornice (SOR-OD on the top and GEOWN on the bottom)

cloud allows the classification algorithm to estimate precisely the true ground geometric characteristics. The quality of these results is made possible by the preservation of the point cloud density by the GEOWN denoising tool. If noisy points were deleted from the point cloud instead of being moved towards their most probable position, the ground detection could not be performed with the same resolution. A point cloud with a preserved density results in a finer description of smooth surfaces.

Point cloud denoising significantly improves ground segmentation performance. This is an example of how the usage of denoising in a classification workflow may help to produce cleaner results. Many algorithms based on surface reconstruction or surface feature estimation, such as meshing or volume computation, could see their performances enhanced by using a denoised point cloud in input rather than a raw point cloud.

\section{CONCLUSION}

The GEOWN denoising tool is efficient, capable and adapted for noisy point cloud enhancement. The GEOWN method was compared to a classical method, and the results show that our algorithm provides more accurate results and a greater noise reduction for similar parameters. The GEOWN denoising tool delivers cleaned point clouds, considering their a priori uncertainties for the estimation of the denoised positions.

The GEOWN denoising tool can be used in several contexts but has a constant objective: extract reliable information from a noisy point cloud. The reliability of the extracted information is linked with the quality of the geometric descriptors that are used. The results presented in this document confirm the quality of the geometrical properties of our algorithm.

The GEOWN denoising method can help point cloud producers and users who require precise data, but are not equipped with high grade LiDAR systems. The tool has point cloud
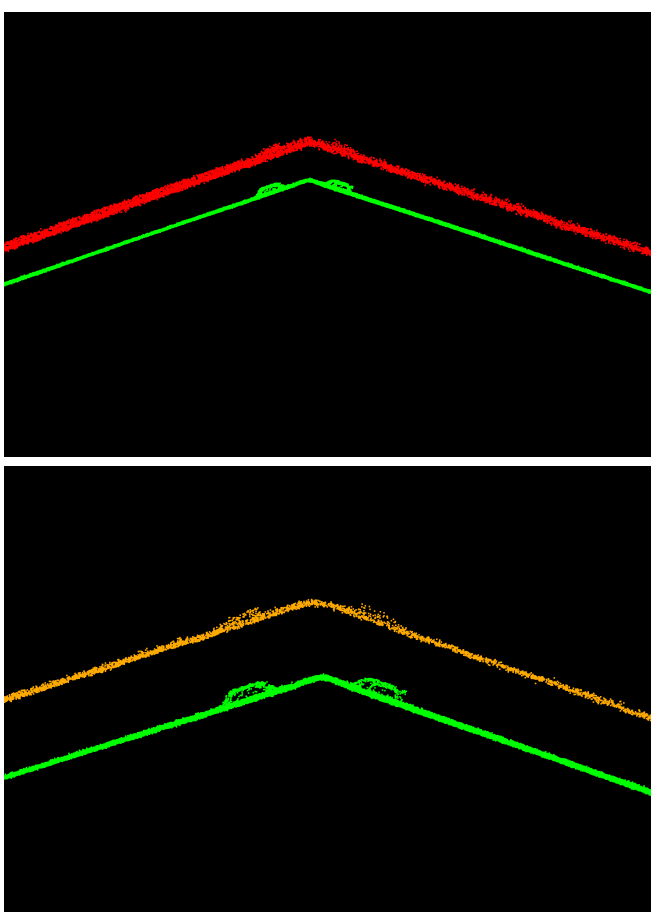

Figure 12. Top: Original point clouds by a low precision LiDAR (red) and a high precision LiDAR (green). Bottom: Denoised point cloud (Orange: denoised point cloud, green: high grade point cloud).

thinning capabilities without excessive smoothing of the sharp edges or features. Even if denoising can't improve the LiDAR resolution, the GEOWN denoising tool can produce clean point clouds with a precision level similar to what could be obtained with a high precision sensor.

Finally, denoising may improve the quality of point cloud classification tools in providing a cleaner description of the surfaces to be denoised. As our denoising tool does not affect either the density nor the complex structures like vegetation, the signal to noise improvement of the point cloud enable the user to get enhanced performances for various applications.

\section{ACKNOWLEDGEMENTS}

We would like to thanks the Company Microdrones Canada Inc. for providing us the point clouds we analysed in this paper, and in particular Stefanie Van-Wierts for her support and relevant comments on this paper. The comparison between the low-cost SICK-LiDAR and the Riegl VUX-UAV was possible thnaks to Microdrones ability to operate the two systems on the same day and flight configuration, using the mdLiDAR 1000 and mdLiDAR 3000 UAV systems.

\section{REFERENCES}

Boulch, A., Marlet, R., 2012. Fast and robust normal estimation for point clouds with sharp features. Eurographics Symp. on Geomery Processing.

Deschaud, J.-E., Goulette, F., 2010. Point cloud non local denoising using local surface descriptor similarity. M. C. T. O. Paparoditis N., Pierrot-Deseilligny M. (ed.), IAPRS, XXXVIII, Part 3A. 

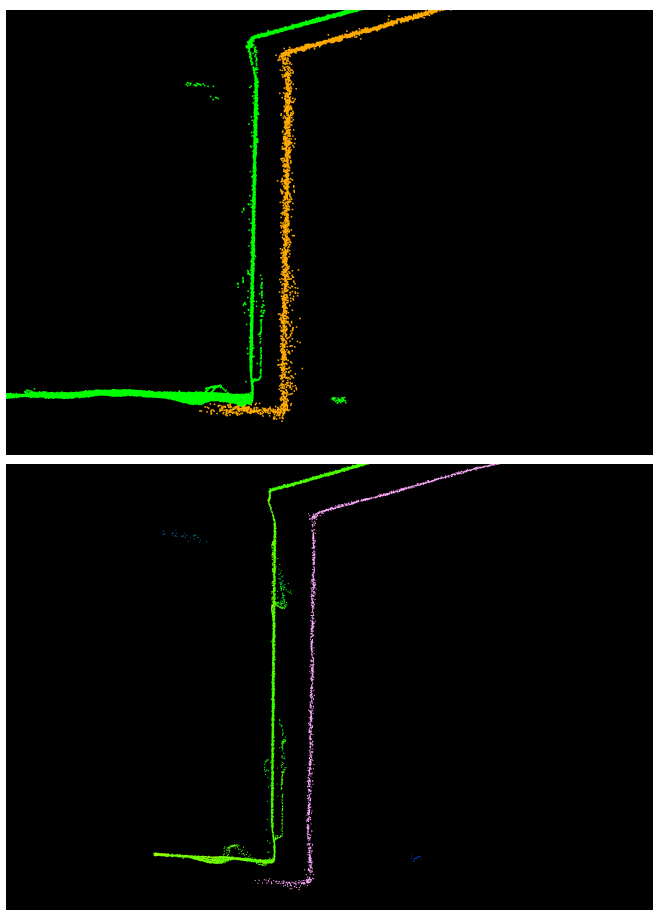

Figure 13. Top: Denoising result $(\mathrm{r}=25 \mathrm{~cm})$ compared to precise LiDAR point cloud (orange: denoised point cloud, green : clean point cloud). Bottom: Denoising result $(\mathrm{r}=40 \mathrm{~cm})$ compared to precise LiDAR point cloud (rose: denoised point cloud, green: clean point cloud)

Digne, J., 2012. Similarity based filtering of point clouds. Proc. of the 2012 IEEE Computer Society Conference on Computer Vision and Pattern Recognition Workshop.

Duguet, F., Durand, F., G.Drettakis, 2004. Robust higher-order filtering of points. Technical report, INRIA.

Fleishman, S., Cohen-Or, D., Silta, C., 2005. Robust moving least-squares fitting with sharp features. Proc. of SIGGRAPH '05, 544-552.

Mitra, N., Nguyen, A., 2003. Estimating surface normals in noisy points clouds data. Proc. of the SoCG'03, San Diego, USA.

Rosmanand, G., Dubrovina, A., R.Kimmel, 2013. Patch Collaborative Spectral Point Cloud Denoising. Computer Graphics Forum, 32, 1-12.

Revised Oct 2019
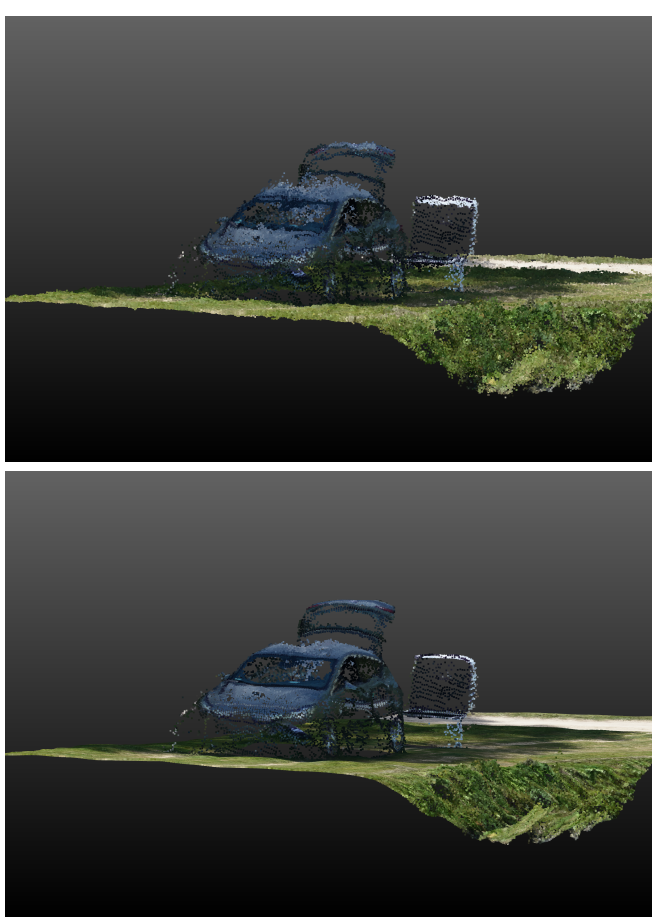

Figure 14. Top: Original dense colorized point cloud as produced by the GEOWN FORMap photogrammetry tool. Bottom: Denoised point cloud.
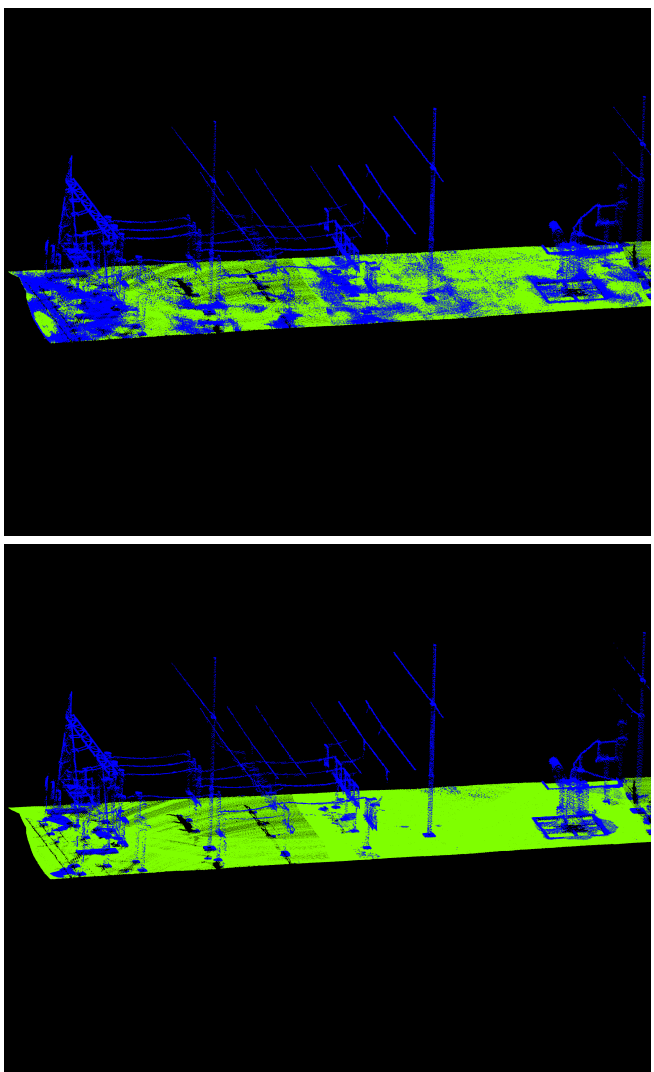

Figure 15. Top: Ground classification on original point cloud (green : ground, blue : not ground). Bottom: Ground classification on denoised point cloud (green : ground, blue : not ground). 